DOI: 10.15593/RZhBiomeh/2016.2.06

УДК 531/534:[57+61]

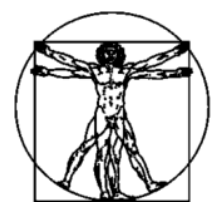

Росеийекий Журнал Биомеханики

www.biomech.ru

\title{
АНАЛИЗ ОСНОВНЫХ ПРИЧИН НЕСРАЩЕНИЙ И СПОСОБОВ ОСТЕОСИНТЕЗА ПЕРЕЛОМОВ ШЕЙКИ БЕДРА
}

\author{
А.Ю. Акулич ${ }^{1}$, Ю.В. Акулич ${ }^{2}$, А.С. Денисов ${ }^{3}$, Д.В. Иванов ${ }^{4}$, А.Ф. Шулятьев ${ }^{2}$ \\ ${ }^{1}$ Клиника травматологии и ортопедии Пермского государственного медицинского университета им. \\ академика Е.А. Вагнера, Россия, 614096, Пермь, ул. Братьев Игнатовых, 2, e-mail: akulichanton@yandex.ru \\ ${ }^{2}$ Кафедра теоретической механики и биомеханики Пермского национального исследовательского \\ политехнического университета, Россия, 614990, Пермь, Комсомольский проспект, 29, e-mail: \\ shulyatev.af@gmail.com \\ ${ }^{3}$ Кафедра травматологии, ортопедии и военно-полевой хирургии Пермского государственного \\ медицинского университета им. академика Е.А. Вагнера, Россия, 614990, Пермь, ул. Петропавловская, 26, \\ e-mail: rector@psma.com \\ ${ }^{4}$ Кафедра математической теории упругости и биомеханики Саратовского национального \\ исследовательского государственного университета имени Н.Г. Чернышевского, Россия, Саратов, 410012 , \\ ул. Астраханская, 83, e-mail: ivanovdv@gmail.com
}

\begin{abstract}
Аннотация. Число несращений и других осложнений при остеосинтезе переломов шейки бедра по-прежнему остается на высоком уровне и достигает 10-30\% от общего числа оперативных вмешательств. Снижение числа осложнений, в первую очередь несращений отломков, является актуальной проблемой, что стимулирует исследования по данной тематике. Целью настоящей работы является критический анализ научной информации о причинах несращений и клинического опыта применения различных способов остеосинтеза как основы создания биомеханических моделей, необходимых для совершенствования методики хирургического лечения переломов шейки бедра. В представленном обзоре рассматриваются публикации за полувековой период (1965-2015-е годы). В большинстве работ этого периода исследуется остеосинтез резьбовыми фиксаторами с диафизарными накладными пластинами и без них, который обеспечивает различную жесткость системы «фиксатор-кость». Существуют различные мнения о причинах несращений. Среди них наиболее обоснованными являются: 1) недостаточное кровоснабжение отломков при идеальной или близкой к ней репозиции; 2) нестабильность фиксации отломков, возникающая вследствие перенапряжения и резорбции кости под витками резьбы при чрезмерном закручивании фиксаторов. В публикациях, посвященных способам остеосинтеза, рассматриваются в основном два вида существующих оперативных вмешательств, различающихся сроками осевой нагрузки на оперированную конечность. Согласно методике 1 система «фиксаторы-кость» подвергается нагрузкам, приближающимся к физиологическим. Методика 2 предполагает движение больного в течение всего периода сращения без опоры на оперированную конечность. При этом наблюдаются лучшие условия сращения.
\end{abstract}

Ключевые слова: перелом шейки бедра, остеосинтез, причины несращений, способы остеосинтеза.

(С Акулич А.Ю., Акулич Ю.В., Денисов А.С., Иванов Д.В., Шулятьев А.Ф., 2016

Акулич Антон Юрьевич, к.м.н., травматолог клиники травматологии и ортопедии, Пермь

Акулич Юрий Владимирович, д.ф.-м.н., профессор кафедры теоретической механики и биомеханики,

Пермь

Денисов Александр Сергеевич, д.м.н., завкафедрой травматологии, ортопедии и военно-полевой

хирургии, Пермь

Иванов Дмитрий Валерьевич, к.ф.-м.н., доцент кафедры математической теории упругости

и биомеханики, Саратов

Шулятьев Андрей Федорович, аспирант кафедры теоретической механики и биомеханики, Пермь 


\section{ВВЕДЕНИЕ}

В России, как и во многих других цивилизованных странах, растет число людей зрелого, пожилого и старческого возраста. В связи с этим увеличивается число лиц с переломами проксимального отдела бедра. Более того, свыше $20 \%$ пострадавших с переломами шейки бедра - это люди молодого и среднего возраста [20, 21], которым вряд ли целесообразно эндопротезирование. Если переломы проксимального отдела бедра составляют до 70 \% всех переломов бедра [6], то на долю переломов шейки бедра из них приходится 50-55 \% [4].

В настоящее время на основании полувековой клинической практики считается, что при лечении описанных переломов наиболее важным фактором является стабильная фиксация отломков для обеспечения максимально быстрого заживления и активизации больного после операции $[3,8]$. Стабильной считается фиксация, при которой отсутствует относительное смещение контактирующих поверхностей отломков. Вместе с тем число несращений и других осложнений остается довольно большим и практически не уменьшилось по сравнению с прошлыми десятилетиями [28]. Поэтому проблема снижения числа несращений является актуальной, что стимулирует продолжение исследований. Накопленные знания причин несращений и клинический опыт применения различных способов остеосинтеза являются базовой информацией при создании биомеханических моделей, необходимых для совершенствования методики хирургического лечения не только перелома шейки бедра, но и других внутрисуставных травм. Целью настоящего исследования являются представление и критический анализ данной информации.

В представленном обзоре рассматриваются публикации за период с 1965 по 2015 год. Во всех работах этого периода исследуется остеосинтез резьбовыми фиксаторами и фиксаторами с различными диафизарными накладками, обеспечивающими достаточную жесткость системы «фиксатор-кость» при минимальной травматичности костной ткани.

\section{ПРИЧИНЫ НЕСРАЩЕНИЙ ОТЛОМКОВ ПОСЛЕ ОСТЕОСИНТЕЗА ШЕЙКИ БЕДРА}

Следует заметить, что анализу подвергались те случаи, когда в экстренном порядке удавалось достигнуть идеальной или близкой к ней репозиции отломков.

Считается, что к несращениям отломков и некрозу костной ткани в области головки и шейки бедра приводит недостаточное их кровоснабжение. Эта гипотеза получила распространение в 60-80-е годы прошлого столетия на основании обнаруженного участия клеточных элементов кровеносных капилляров непосредственно в репаративной регенерации костной ткани $[9,17,26,27]$. Поэтому в зарубежной практике в те годы «золотым стандартом» при остеосинтезе переломов шейки бедра являлось максимально быстрое и надежное восстановление кровоснабжения головки и шейки бедра [18]. Особенно этот стандарт учитывался при лечении больных активной возрастной группы, поскольку они, как правило, подвергаются высокоэнергетическим травмам с осевым смещением отломков, затрудняющим кровоснабжение.

Количественное обоснование этого предположения было получено в начале XXI века. Так, клинический анализ 550 переломов шейки бедра больных в возрасте 15-50 лет, произошедших в США между 1976 и 2003 годами, показал, что переломы со смещением наблюдались существенно чаще (в 80 \% случаев). Асептический некроз головки бедра в этих случаях развивался в четыре раза чаще, чем при травмах без смещения отломков [12]. Кроме того, авторы сделали важный вывод для клинической практики о допустимом времени откладывания операции. 
Статистические исследования выборки из 170 травм со смещениями не установили корреляцию между числом несращений и временем задержки операции в интервале 12-48 часов. Спустя 48-72 часа после травмы асептический некроз запускается уже автоматически, несмотря на репозицию и фиксацию отломков.

Другой негативный фактор, влияющий на вероятность возникновения осложнений, - это нестабильность фиксации отломков вследствие резорбции костной ткани под опорной поверхностью резьбы фиксаторов, наблюдающаяся в первые две недели после оперативного вмешательства [2]. При этом предполагается, что причиной резорбции является превышение предельно допустимых значений напряжений сжатия («напряжений непереносимости» 7 МПа [5]) в костной ткани под витками резьбы, вызванное неконтролируемой величиной момента закручивания фиксатора. Клинические исследования результатов остеосинтеза с контролируемой величиной момента закручивания фиксатора подтвердили это предположение [1].

Gefen [14] также полагает, что нестабильность фиксаторов, возникающая вследствие резорбции костной ткани вокруг имплантата, является причиной несращений переломов. Однако механизм резорбции он объясняет тем, что металлические имплантаты имеют существенно большую жесткость (более чем в 100 раз), чем кость, и вследствие этого «вся нагрузка передается на фиксатор, что вызывает атрофию прилегающей к фиксатору кости в ответ на снижение действующей на нее механической нагрузки». Это рассуждение представляется ошибочным. Поскольку нагрузка на фиксатор, погруженный в костную ткань, передается костной тканью, то в силу постулата механики - действие равно противодействию, костная ткань в каждой точке контакта с фиксатором испытывает такие же нагрузки, как и фиксатор. То есть биомеханические условия для атрофии кости вокруг фиксатора отсутствуют. Этот вывод подтверждается результатами компьютерного моделирования остеосинтеза перелома шейки бедра [10].

\section{СПОСОБЫ ОСТЕОСИНТЕЗА ПЕРЕЛОМОВ ШЕЙКИ БЕДРА}

В данном разделе рассматриваются работы, показывающие преимущества того или иного способа фиксации отломков, обеспечивающего достаточную, с точки зрения биомеханики, стабильность фиксации перелома.

Для оценки способов фиксации авторами цитируемых работ применялись методы математического и численного моделирования, а также натурные испытания сочленений фиксаторами трупных и искусственных костей на универсальных испытательных машинах. Рассматривались различные длины резьбовых фиксаторов, осуществлялось сравнение их с имплантатами других типов, а также исследовались варианты и конфигурации установки фиксаторов в кость.

При выборе источников для обзора предпочтение отдавалось статьям, опубликованным в последнее десятилетие в зарубежных и отечественных высокорейтинговых изданиях. Поиск статей осуществлялся также в электронных библиотеках eLIBRARY, Medline и др.

Несмотря на то что проксимальные блокирующие пластинки показывают более высокие результаты по стабильности фиксации, они являются статическими имплантатами и не позволяют создавать необходимую компрессию на стыке отломков [13]. Этого недостатка лишены резьбовые фиксаторы, создающие необходимую компрессию отломков путем упругого деформирования костной ткани при закручивании. В связи с этим далее анализируются способы внутренней фиксации резьбовыми фиксаторами. 


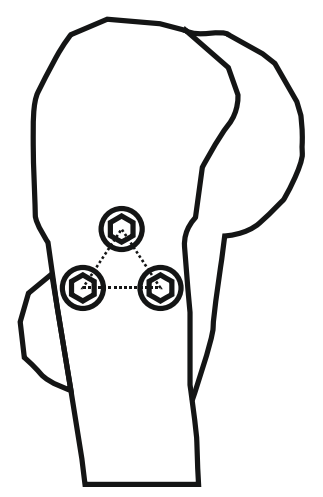

$a$

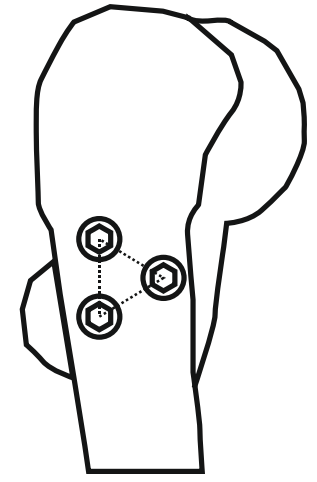

6

Рис. 1. Схемы установки фиксаторов: a) горизонтальная; б) вертикальная

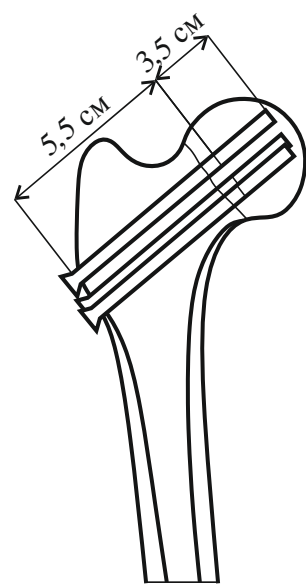

$a$

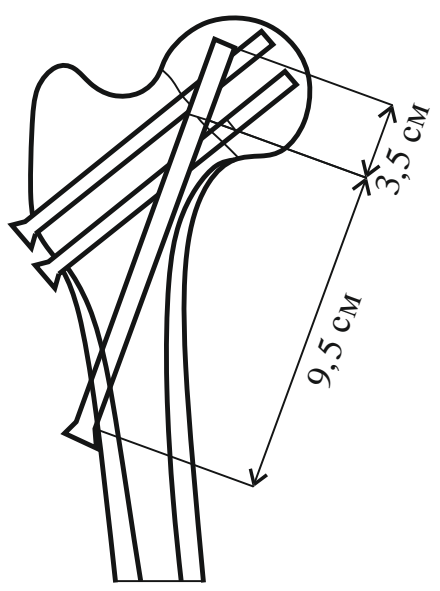

$\sigma$

Рис. 2. Схемы традиционного (a) и нового (б) способов установки фиксаторов

В работе [19] авторы сравнивали две конфигурации установки резьбовых фиксаторов, расположенных по треугольным схемам (рис. 1). В эксперименте на искусственных костях на головку бедренной кости прессом вдавливали сферическую чашу. Разрушающую нагрузку и структурную жесткость определяли из соответствующих графиков «нагрузка-перемещение».

Авторы сделали вывод, что установка двух резьбовых фиксаторов на одном уровне, как в горизонтальной схеме, приводит к потере стабильности фиксации.

Аналогичные выводы были сделаны после выполнения натурных испытаний на конструктивную жесткость системы «фиксаторы-кость» и в работе Selvan и др. [25]: треугольная конфигурация в отличие от вертикально расположенных двух или трех фиксаторов оказалась более стабильной и прочной.

Oakey с соавт. [22] показал, что треугольная конфигурация с одним нижним фиксатором считается более надежной, чем конфигурация с одним верхним фиксатором.

Filippov [13] предложил новый способ установки трех фиксаторов (рис. 2).

Идея здесь заключается в том, что нижний фиксатор предлагается устанавливать под большим углом и непараллельно другим фиксаторам и оси головки бедра. При таком способе нижний фиксатор устанавливается в месте, где кортикальный слой 

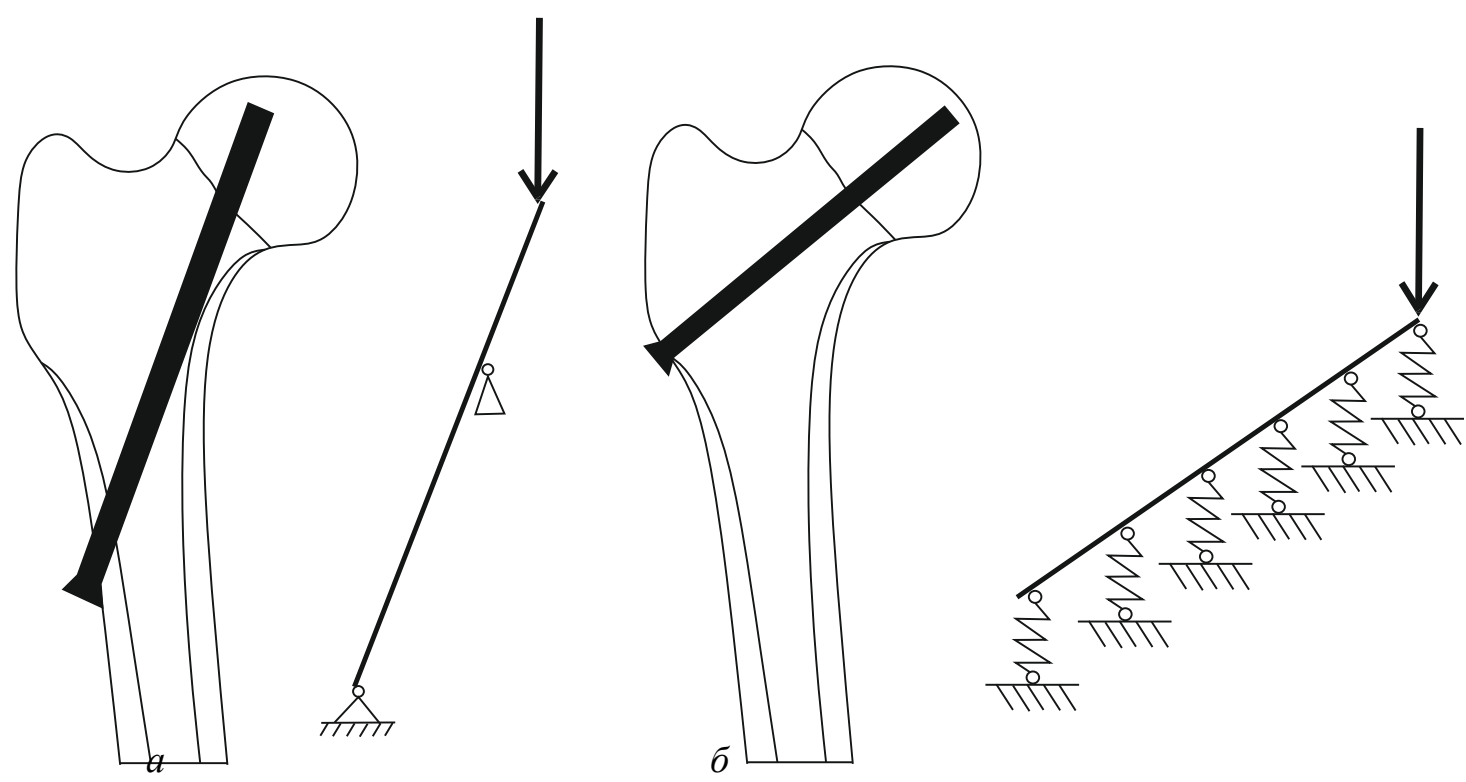

Рис. 3. Нагрузки на нижний фиксатор в традиционной (a) и новой (б) схемах установки

кости является более толстым, а фиксатор работает как балка, подкрепленная двумя шарнирами (рис. 3,б). При традиционном способе установки нижний фиксатор работает как балка, подкрепленная по всей длине упругим слоем (рис. $3, a$ ). Клинические результаты показали превосходство нового способа установки нижнего фиксатора как более жесткого по сравнению с традиционным способом. Однако следует отметить, что непараллельность фиксаторов не позволяет мышцам сустава осуществлять при рефлекторном сокращении сжатие отломков, необходимое для стабильности фиксации.

В работе [24] приводятся результаты натурных и численных экспериментов трех систем фиксации переломов шейки бедра: треугольная конфигурация из стягивающих фиксаторов, проксимальная блокирующая пластина и динамический стержень с антиротационным винтом. Исследовались статические нагружения кости с установленными фиксаторами с учетом постоянных изотропных и однородных механических характеристик костной ткани. Три фиксатора показали себя хуже двух других рассмотренных имплантатов: были отмечены наименьшая стабильность фиксации, а также большие перемещения головки бедра. Эти данные подтверждаются как натурными, так и численными экспериментами. Результаты и выводы работы [24] кажутся очевидными, так как в этом случае система, состоящая из трех сжимающих фиксаторов, является заведомо менее жесткой по сравнению с двумя другими рассмотренными системами. На самом деле две другие системы имеют не только жесткую и прочную пластину, но и как минимум четыре винта.

Несмотря на то что проксимальные блокируемые пластины показывают более высокие результаты по стабильности фиксации, они являются статическими имплантатами и не позволяют создавать необходимую компрессию на стыке отломков [22]. Этого недостатка лишены резьбовые фиксаторы.

Численное конечно-элементное моделирование остеосинтеза перелома шейки бедра различными конфигурациями стягивающих фиксаторов проведено Gok c соавт. [15]. Сравнивались треугольные конфигурации, вертикальное расположение двух и трех фиксаторов, а также четырехугольная конфигурация (рис. 4). 


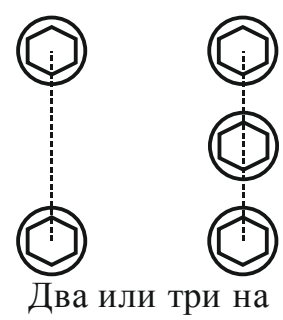
одной линии

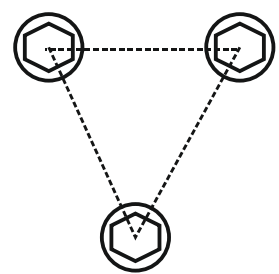

Обращенный треугольник

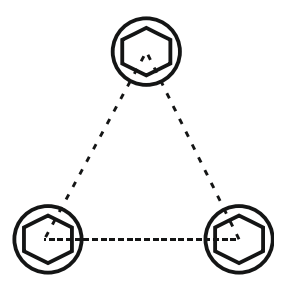

Треугольник

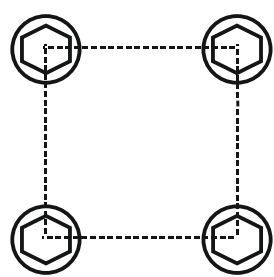

Квадрат

Рис. 4. Конфигурации стягивающих винтов, исследованные Gok и соавт. [15]

Система «кость-фиксаторы» нагружалась статической нагрузкой $350 \mathrm{H}$, рассматривались задачи о контактном взаимодействии фиксаторов и костных отломков. Костная ткань рассматривалась как изотропный и однородный материал с постоянными модулями упругости губчатой и кортикальной костной ткани. Треугольная конфигурация фиксаторов с двумя нижними параллельными фиксаторами показала себя лучше других с точки зрения жесткости конструкции. Максимальные напряжения на фиксаторах и кости в случае треугольной конфигурации оказались существенно ниже (от 2 до 10 раз), чем напряжения на фиксаторах и костных отломках при других конфигурациях. Здесь стоит отдельно сказать о нагрузке на систему «костьфиксаторы», так как выбор усилий, прикладываемых к кости, также является предметом дискуссии.

По нашему мнению, в первое время послеоперационного периода основным усилием, которое необходимо моделировать, является сжимающая нагрузка от фиксаторов. Более того, больному в первые недели после остеосинтеза запрещено опираться на поврежденную конечность, а вопрос дозирования нагрузки на оперированную конечность остается открытым [7].

Сравнивалась [11] фиксация перелома шейки бедра двумя параллельными фиксаторами с резьбой на конце, двумя параллельными фиксаторами с резьбой от начала до конца и двумя непараллельными фиксаторами с резьбой на конце. Натурные испытания проводились для синтетических костей (21 штука). В месте перелома удалялся участок толщиной 4 мм, а имплантаты испытывались на способность сопротивления укорочению кости после остеосинтеза. С точки зрения биомеханики наилучшим образом показала себя первая конфигурация с частичной резьбой на конце и усиленная гидроксиапатитом в месте перелома (пространство между отломками заполнялось гидроксиапатитом). Жесткость системы «кость-имплантат» была наиболее близка к жесткости нормальной кости без перелома.

В работе [16] численно исследовалась стабильность остеосинтеза перелома шейки бедра резьбовыми фиксаторами разного диаметра $(6,5 ; 7,0 ; 7,3$ мм), устанавливаемыми в треугольной конфигурации. Система «кость-фиксаторы» нагружалась силами, моделирующими подъем пациента по лестнице. Наиболее 
прочными и стабильными оказались фиксаторы диаметром 7,3 мм. Результаты для фиксаторов диаметром 6,5 и 7,0 мм оказались схожими.

Клинические результаты говорят о том, что более трех фиксаторов использовать нецелесообразно ввиду высокой травматичности операции [15].

\section{ЗАКЛЮЧЕНИЕ}

Важно отметить, что в тех цитируемых работах, в которых оценка способа фиксации осуществлется без указания нагрузок на конечность в период послеоперационного сращения (первые 4-6 месяцев), для создания выгодных условий для сращения используется ранняя нагрузка конечности весом тела в первые дни после операции. Этот подход имеет недостаток, связанный с повышенным риском нарушения стабильности контакта между отломками ввиду повышенной динамической нагрузки при ходьбе и случайных перегрузок больной конечности. При этом возможно периодическое изменение величины зазора между отломками, превышающее диаметр одной клетки (1-3 мкм), которое вызывает необратимую резорбцию костной ткани контактирующих поверхностей [28] и, как следствие, несращение перелома [8].

Альтернативная методика лечения, используемая, например, в ряде клиник России, состоит в том, что в период сращения отломков больной перемещается на костылях, а больная конечность иммобилизируется с помощью бандажа. Очевидно, что риск нарушения стабильности контакта между отломками в этом случае минимален. Кроме того, проксимальный отдел бедра в этом положении больной конечности рефлекторно нагружается усилиями мышц сустава, действующими вдоль оси шейки. Поскольку оси фиксаторов располагаются также параллельно оси шейки бедра, то создается дополнительная компрессия отломков, способствующая стабильности фиксации.

\section{СПИСОК ЛИТЕРАТУРЫ}

1. Акулич А.Ю., Акулич Ю.В., Денисов А.С. Возможности аргументированного подхода к остеосинтезу шейки бедра резьбовыми фиксаторами с учетом механических и адаптационных свойств костной ткани // Гений ортопедии. - 2012. - № 2. - С. 22-26.

2. Акулич Ю.В., Акулич А.Ю., Денисов А.С. Индивидуальный остеосинтез шейки бедра резьбовыми фиксаторами // Российский журнал биомеханики. - 2010. - Т. 14, № 1. - С. 7-16.

3. Войтович А.В. Оперативное лечение больных с переломами проксимального отдела бедра в системе медицинской реабилитации: автореф. дис. ... д-ра мед. наук. - СПб., 1994. - 32 с.

4. Грицанов А.И. Оценка устройств, обеспечивающих стабильность фиксации отломков // Материалы междунар. конгресса «Человек и его здоровье». - СПб., 1997. - С. 245.

5. Джанг Д.-Й., Цуцуми С., Канг Й.-Б., Секель Р. Численное прогнозирование перемещения вертлужной чаши под действием высоких сжимающих напряжений на основе моделирования резорбции костной ткани // Российский журнал биомеханики. - 2005. - Т. 9, № 3. - С. 32-45.

6. Каплан А.В. Достижения и перспективы гериатрической травматологии // Ортопедия, травматология и протезирование. - 1983. - № 2. - С. 10-15.

7. Мельцер Р.И., Иванов Д.В., Лозовик И.П., Верховод А.Ю., Поченты Д.О. Послеоперационное ведение больных с неопорными переломами костей голени в условиях контролируемой осевой нагрузки // Ученые записки Петрозаводского государственного университета. Серия: Естественные и технические науки. - 2013. - № 8 (137). - С. 37-39.

8. Мюллер М.Е., Алльговер М., Шнейдер Р., Виллингер Х. Руководство по внутреннему остеосинтезу // M.: AdMarginem, 1996.

9. Суслов Е.Н. Возрастные особенности микроциркуляции // Ультраструктура микроциркуляторных путей в патологии. - Львов, 1974. - С. 5-11.

10. Шулятьев А.Ф., Акулич Ю.В., Акулич А.Ю., ДенисовА.С. 3D-моделирование остеосинтеза перелома шейки бедра // Материалы рос. конф. с междунар. участием «Экспериментальная и компьютерная биомедицина». - Екатеринбург, 2016. - С. 37. 
11. Alves T., Neal J.W., Weinhold P.S., Dahners L.E. Biomechanical comparison of 3 possible fixation strategies to resist femoral neck shortening after fracture // Orthopedics. - 2010. - Vol. 33, № 4. P. 233- 237.

12. Damany D.S., Parker M.J., Chojnowski A. Complications after intracapsular hip fractures in young adults. Meta-analysis of 18 published studies involving 564 fractures // Injury. - 2005. - Vol. 36. - P. 131-141.

13. FilipovO. Biomechanics of the fractured femoral neck. The new BDSF-method of positioning the implant as a simple beam with an overhanging end // Injury and Skeletal Biomechanics / Ed. T. Goswami. - Rijeka: InTech, 2012. DOI: 10.5772/47839.

14. Gefen A. Optimizing the biomechanical compatibility of orthopedic screws for bone fracture fixation // Med. Eng. Phys. - 2002. - Vol. 24, № 5. - P. 337-347.

15. Gok K.,Inal S. Biomechanical comparison using finite element analysis of different screw configurations in the fixation of femoral neck fractures // Mech. Sci. - 2015. - Vol. 6. - P. 173-179.

16. Ishak M.F., Kadir M.R.A., Rahman S.Ab. The effect of cannulated hip screw size in the treatment of femoral neck fracture // JournalTeknologi. - 2015. - Vol. 77, № 7. - P. 13-17.

17. KrompecherS. Local tissue metabolism and the quality of the callux // Calluxformation. - Budapest, 1967. P. 275-300.

18. Kuzyk P.R.T., Schemitsch E.H. Intracapsular femoral neck fracture: how does delay in surgery affect complication rate? // Wright J.G. Evidence-based orthopedics: the best answers to clinical questions Philadelphia: Saunders Co., 2009. - P. 396-400.

19. Lichtblau S., Gallina J., Nasser P., Munyoki M., Jepsen K. A biomechanical comparison of two patterns of screw insertion // Bull. NYU Hosp. Jt Dis. - 2008. - Vol. 66, № 4. - P. 269-271.

20. Liporace F., Gaines R., Collinge C., Haidukewych G.J. Results of internal fixation of pauwels type-3 vertical femoral neck fractures // Journal of Bone \& Joint Surgery. - 2008. - Vol. 90. - P. 1654-1659.

21. Ly T.V., Swiontkowski M.F. Treatment of femoral neck fractures in young adults // Journal of Bone and Joint Surgery. American Volume. - 2008. - Vol. 90. - P. 2254-2266.

22. Oakey J.W., Stover M.D., Summers H.D., Sartori M., Havey R.M., Patwardhan A.G. Does screw configuration affect subtrochanteric fracture after femoral neck fixation? // Clinical Orthopaedics and Related Research. - 2006. - Vol. 443. - P. 302-306.

23. Perren S.M., Ganz R., Rüter A. Oberflächliche Knochenresorption um Implantate // Med.-Orthop. Tech. 1975. - Vol. 95. - P. 6-10.

24. Samsami S., Saberi S., Sadighi S., Rouhi G. Comparison of three fixation methods for femoral neck fracture in young adults: Experimental and numerical investigations // Journal of Medical and Biological Engineering. - 2015. - Vol. 35, № 5. - P. 566-579.

25. Selvan V.T., Oakley M.J., Rangan A., Al-Lami M.K. Optimum configuration of cannulated hip screws for the fixation of intracapsular hip fractures: a biomechanical study // Injury. - 2004. - Vol. 35, № 2. P. 136-141.

26. Trueta J. Bloodsupply and the rate healing of tibial fractures // Clin. Orthop. - 1974. - Vol. 105. P. 11-26.

27. Urist M.R., Lange R.J., Finerman G.A. Bone cell differentiation and growth factors // Science. - 1983. Vol. 220. - P. 420-429.

28. Wu C.C. Using biomechanics to improve the surgical technique for internal fixation of intracapsular femoral neck fractures // Chang. Gung.Med. J. - 2010. - Vol. 33, № 3. - P. 241-251.

\title{
ANALYSIS OF MAIN REASONS OF NONUNIONS AND METHODS OF OSTEOSYNTHESIS OF THE FEMORAL NECK FRACTURES
}

\author{
A.Yu. Akulich, Yu.V. Akulich, A.S. Denisov (Perm, Russia), \\ D.V. Ivanov (Saratov, Russia), A.F. Shulyatev (Perm, Russia)
}

The number of nonunions and other complications of osteosynthesis of femoral neck fractures remains at a high level, reaching 10-30\% of the total number of surgical interventions. Reducing the number of complications, primarily nonunions of bone fragments, is an urgent problem that encourages research on the subject. The purpose of this paper is a critical analysis of the scientific reasons of nonunions and clinical experience of the use of different methods of osteosynthesis as the basis for the creation of biomechanical models 
needed to improve methods of surgical treatment of hip fractures. This review considers publications of the past half-century (1965-2015 years). In most works of this period, osteosynthesis with locking screws with diaphyseal patch plates and without them was studied. There are different views about the causes of nonunions. Among them, the most reasonable are: 1) lack of blood supply to the bone fragments at the ideal reposition; 2) unstable fixation of bone fragments arising as a result in overstress and resorption of the bone at the threads zones. In publications devoted to osteosynthesis methods, basically two kinds of surgical interventions were considered. According to the first method,"bone-implant" systemare loaded under physiological conditions. Second technique involves movement of the patient during healing period without bearing on the operated limb. The second method provides apparently best healing conditions.

Key words: femoral neck fracture, osteosynthesis, reasons of nonunions, methods of osteosynthesis. 